\title{
Evaluation of Measurement Uncertainty Based on Monte Carlo Method
}

\author{
X M Wang, J L Xiong and J Z Xie \\ School of Intelligent Science, National University of Defense Technology, Changsha, China
}

\begin{abstract}
The developments of scientific technology are inseparable from various measurement methods. Measurement uncertainty is an indication that evaluates the credibility of the measurement results directly, and it affects the development of technology and economy indirectly. Monte Carlo method (MCM) is an effective method to evaluate the measurement uncertainty, because it can evaluate the measurement uncertainty in the complex model and environment. The application range of MCM is large than the traditional method that recommended in the "Guide to the Uncertainty in Measurement (GUM)". Based on the study of Monte Carlo method, this paper establishes a model for MCM to evaluate the uncertainty of measurement. Finally, as an example, we use the MCM to evaluate the measurement uncertainty of the six-and-a-half digital multimeter (DMM), which verifies the validity of MCM for evaluating the measurement uncertainty.
\end{abstract}

\section{Introduction}

The measurement uncertainty is the uncertainty and change of measurement result. It is an estimation that the true value of measured value is in a certain range. And it is a parameter included in the measurement result to indicate the dispersion of the measured value. The definition of measurement uncertainty shows that a complete measurement result should contain both the measured value and the measurement uncertainty[1]. The measurement uncertainty is usually assessed by the mathematical model of the measurement process and the uncertainty transmission rate. The method used in the field of measurement uncertainty assessment is GUM, which is mainly derived from ISO / IEC GUIDE 98-3: 2008 "Measurement Uncertainty-Part 3: Measurement Uncertainty Representation Guide"[2]. On the basis of this international standard and JJF 1059-1999, China issued the new edition JJF 1059.1-2012 "Evaluation and Expression of Measurement in Uncertainty" on December 3th, 2012, and it implemented on June 3th, 2013[3]. The GUM obtains the measurement uncertainty of measurand through the relationship between input and output, and using the uncertainty transmission rate of input optimal estimation and its associated standard uncertainty. However, the application of GUM has some limitations and assumptions[4]. When the probability distribution of input should be distributed symmetrically, such as normal distribution and t-distribution, and the measurement model is a linear model or can be converted into a linear model, the GUM can be used to assess the measurement uncertainty.

MCM is a numerical method to achieve distributed distribution by repeated sampling. MCM obtains the discrete sampling values of measurand $Y$ through sampling the input $X_{i}$ according to its probability density function (PDF) and selecting the transmission model of the input distribution according to the measurement model. Finally, obtain the optimal estimation, the standard uncertainty and the inclusion interval of measurand $Y$ through the calculated discrete sampling values $Y[5]$. MCM can be applied in a wide range, in addition to the scope of the traditional GUM. What's more, the application range suitable for MCM: the scale of the uncertainty component is not approximate; the deviation of measurement model is difficult to obtain; the PDF of measurand is not a normal distribution or a $\mathrm{t}$ distribution; the estimated value of measurand and its standard uncertainty are equivalent; the measurement model is complex or non-linear; the PDF of input is asymmetric[6]. When the conditions required by the GUM are not or not sure satisfied, and the GUM is difficult to calculate measurement uncertainty due to the complexity of the model, the MCM is all suitable to assess the measurement uncertainty. In this paper, we use the MCM to evaluate the measurement uncertainty of the six-and-a-half DMM based on the MATLAB platform to get the measurement uncertainty result of DMM.

\section{Measurement uncertainty evaluation using MCM}

\subsection{Measurement uncertainty}

The measurement uncertainty is defined as the parameter that is reasonably assigned to the dispersion of the measured value, which is associated with the measurement result[7][8]. Measurement uncertainty is 
usually assessed by the mathematical model of measurement process and the uncertainty transmission rate. If the relationship between measurand $Y$ and input $X_{1}$, $X_{2}, \ldots, X_{n}$ is $Y=f\left(X_{1}, X_{2}, \ldots, X_{n}\right)$, the measurement uncertainty can be obtained according to the uncertainty transfer diagram shown in Fig. 1, and the synthetic standard uncertainty of $\mathrm{Y}$ can be calculated according to formula (1).

$$
u_{c}(y)=\sqrt{\sum_{i=1}^{N}\left(\frac{\partial f}{\partial x_{i}}\right)^{2} u^{2}\left(x_{i}\right)+2 \sum_{i=1}^{N-1} \sum_{j=1, j \neq i}^{N} \frac{\partial f}{\partial x_{i}} \frac{\partial f}{\partial x_{j}} r\left(x_{i}, x_{j}\right) u\left(x_{i}\right) u\left(x_{j}\right)}
$$

Where $u\left(x_{i}\right)$ is the standard uncertainty of the input $X_{i}$, which can be type A standard uncertainty or type B standard uncertainty. Where $u_{c}(y)$ is the synthetic standard uncertainty of measurand $Y$, which indicates the dispersion of measurand $Y$. Where $\partial f / \partial x_{i}$ is the partial derivative of the function $Y=f\left(X_{1}, X_{2}, \ldots, X_{n}\right), r\left(x_{i}, x_{j}\right)$ is the correlation coefficient of the uncertainty, and $N$ is the total number of the input quantity. If each standard uncertainty of the input $X_{i}$ can be regarded as unrelated, i.e. $r\left(x_{i}, x_{j}\right)=0$, then the synthetic standard uncertainty can be reduced to the formula (2)

$$
u_{c}(y)=\sqrt{\sum_{i=1}^{N}\left(\frac{\partial f}{\partial x_{i}}\right)^{2} u^{2}\left(x_{i}\right)}
$$

If the measurand $Y$ is measured by the measuring device directly, the relationship between measurand and input cannot be expressed by a fixed function. Through the uncertainty analysis, there are $N$ obvious uncertain components $u_{i}$ in the measurement result, and each uncertain component is not correlated. Suppose that each uncertainty component has the same sensitivity degree to the measurement result, the synthetic standard uncertainty can be calculated according to formula (3).

$$
\begin{aligned}
& u_{c}(y)=\sqrt{\sum_{i=1}^{N}\left(u_{i}\right)^{2}} \\
& x_{1} \pm u\left(x_{1}\right) \longrightarrow \\
& x_{2} \pm u\left(x_{2}\right) \longrightarrow \\
& x_{n} \pm u\left(x_{n}\right) \longrightarrow
\end{aligned} Y=f\left(X_{1}, X_{2}, \cdots, X_{n}\right) \longrightarrow y \pm u(y)
$$

Figure 1. Uncertainty transfer diagram

\subsection{Monte Carlo method}

MCM is a statistical test method, which be used to solve the mathematical physics problem, that is difficult to determine the formula, through statistical sampling theory. For a statistic with uncertainty PDF, MCM is easier to solve the synthetic uncertainty which approaches the real solution[4][9]. Unlike the GUM method using the linearized model propagation uncertainty, MCM evaluates measurement uncertainty by a numerical method to achieve distributed propagation.
When the measurement model is linear and the output PDF is a normal distribution, the GUM method can provide accurate uncertainty and inclusion intervals[10]. Fig. 2 depicts the independent input distributions transmission, and the PDF of output quantity $Y$ is obtained through three PDFs of input quantities $X_{1}, X_{2}, X_{3}$ with the model propagation. The three PDFs of input quantities $X_{1}, X_{2}, X_{3}$ in Fig. 2 are normal distributions, triangular distributions and normal distributions respectively, which are independent, and the PDF of output quantity $Y$ is an asymmetry distribution. If the PDF of output quantity $Y$ is asymmetry like in Fig. 2, then the measurement uncertainty evaluation. MCM carries out the discrete sampling of the input quantity PDF, and then calculates the discrete sampling of output quantity $Y$ by the measurement model. Next, the best estimate, the standard uncertainty, and the inclusion interval of the output quantity $Y$ are calculated by the discrete distribution value of the output quantity, which are improved as the number of sample $M$ of the input $X$ increases.

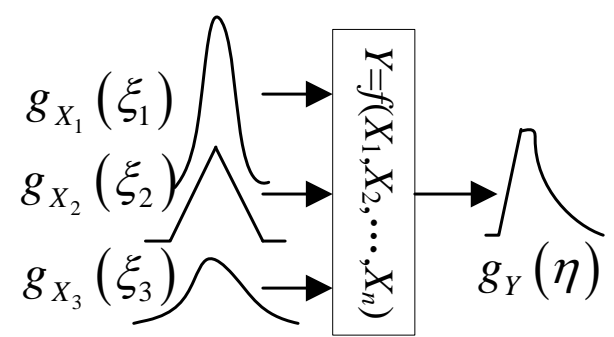

Figure 2. Independent input distributions transmission

\subsection{Measurement uncertainty evaluation using MCM}

The parameter estimation is mainly divided into two kinds, one is that estimate unknown parameters according to the samples when the overall distribution is known and one or several of the parameters is unknown; the other is that the overall distribution and the parameters are unknown. If the overall distribution is known, the commonly methods include moment estimation, maximum likelihood estimation, Bayesian estimation and least squares estimation. The intelligent algorithm is more and more important in parameter estimation, whose principle is to simulate the biological population activities, the information transmission between individuals and individuals. The parameter estimation is mainly divided into two kinds, one is that estimate unknown parameters according to the samples when the overall distribution is known and one or several of the parameters is unknown; the other is that the overall distribution and the parameters are unknown. If the overall distribution is known, the commonly methods include moment estimation, maximum likelihood estimation, Bayesian estimation and least squares estimation. The intelligent algorithm is more and more important in parameter estimation, whose principle is to simulate the biological population activities, the information transmission between individuals and individuals. 
In the measurement uncertainty evaluation using MCM, the mathematical model $Y=f\left(X_{1}, X_{2}, \ldots, X_{n}\right)$ between the input $\left(X_{1}, X_{2}, \ldots, X_{n}\right)$ and the output $Y$ is chosen first according to the physical model, mathematical formula or experience of the measurement. Then determine the distribution and its parameters of the input $\left(X_{1}, X_{2}, \ldots, X_{n}\right)$ quantities uncertainty, which according to generate the various distributed random variables $\xi_{1}, \xi_{2} \ldots, \xi_{n}$. Generally, the various distributed random variables are usually generated by the inverse function of the random variable obeyed uniformly distribution in the interval $(0,1)$. The output value $y_{i}=f\left(\xi_{1}\right.$, $\left.\xi_{2} \ldots, \xi_{n}\right)$ is obtained by putting the random variables $\xi_{1}$, $\xi_{2} \ldots, \xi_{n}$ into the mathematical model $Y=f\left(X_{1}, X_{2}, \ldots, X_{n}\right)$. Since the range of each random variable is distributed in entire domain, the obtained $y_{i}$ may cover all possible values of the output quantity $Y$. The mean value $E_{Y}$ and the standard deviation $S_{Y}$ of the output quantity $Y$ are calculated by the formula (4) and (5) using the $M$ values $y_{1}, y_{2} \ldots, y_{M}$ generated by the MCM.

$$
E_{Y}=\frac{1}{M} \sum_{i=1}^{M} y_{i}
$$

$\left.S_{Y}=\frac{1}{n} \sum_{i=1}^{n}\left(y_{i}-Y\right)^{2} /(n-1)\right]^{1 / 2}$

The specific implementation steps of measurement uncertainty evaluation using MCM are shown in Figure 3, which mainly including MCM input, MCM transmission, MCM output and result report. The MCM input contains the following five aspects: defining the output of $Y$, which is the measurand, determining the input quantity $X=\left(X_{1}\right.$, $\left.X_{2}, \ldots, X_{n}\right)$ associated with the output $Y$, establishing the model $Y=f(X)$ between the output $Y$ and the input $X$, determining the PDF of the each input $X_{i}$ with the information obtained, and selecting the MCM sampling size $M$ which is taken $10^{6}$ generally. The more common distributions of input $X$ are normal distribution, the uniform distribution and so on. The MCM transmission is calculating the corresponding output value $Y_{j}$ for each sample $\left(x_{1 j}, x_{2 j}, \ldots, x_{n j}\right)$ according to the model $Y=f(X)$. The samples $\left(x_{1 j}, x_{2 j}, \ldots, x_{n j}\right)$ are extracted from the PDF of input $X_{i}$,where $i=1,2, \ldots, n$ and $j=1,2, \ldots, M$. The MCM output is mainly sorting these $M$ values of output $Y$ by the strictly ascending order, and getting the discrete representation $G$ of the PDF of the output $Y$ according to the sorted sample values $\left(y_{1}, y_{2}, \ldots, y_{n}\right)$. Finally, according to the discrete representation $G$, the result report is mainly calculating the estimated value $y$ and the standard uncertainty $u(y)$ of the output $Y$, and the inclusion interval $\left[y_{\text {low }}, y_{\text {high }}\right]$ of $Y$ with given inclusion probability $p$.

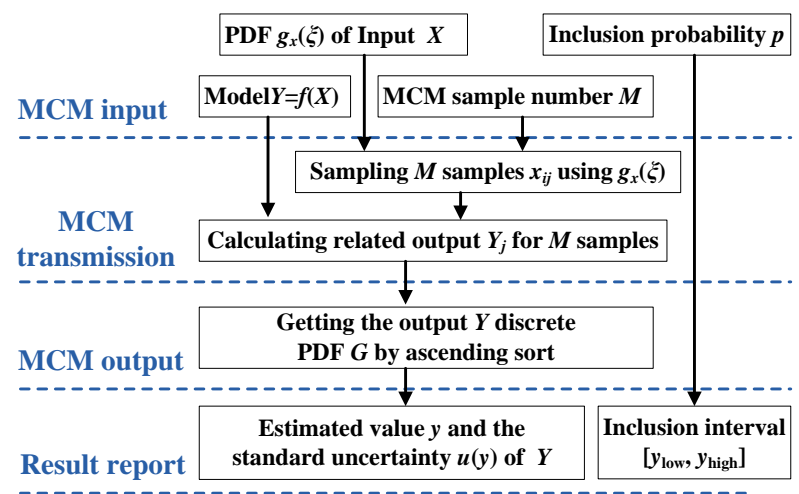

Figure 3. Specific implementation steps of measurement uncertainty evaluation using MCM

\section{MCM for evaluating digital multimeter uncertainty}

Uncertainty evaluation of digital multimeter by MCM, first of all, we need to determine the mathematical model of input and output. The uncertainty source of digital multimeter mainly include: the voltage drift of the multifunction calibrator since the last calibration, the effect of bias, nonlinearity and gain change on the voltage value of the multifunction calibrator instrument, the influence of ambient temperature on the voltage value of the calibrator, the influence of the power supply voltage, and the load effect caused by the limited input impedance of the calibrated DMM. According to the reference [11], the measurement model of DMM is established as shown in formula (6)

$u_{\mathrm{c}}=V_{i}-V_{\mathrm{s}}+\Delta V_{i}-\Delta V_{\mathrm{s}}$

Where $V_{i}$ is the voltage value measured by the DMM, $V_{s}$ is the output voltage of the multifunction calibrator, and these two variables $V_{i}$ and $V_{s}$ are subject to the normal distribution. Where $\Delta V_{i}$ is the effect of the DMM resolution on the measurement result, $\Delta V_{s}$ is the comprehensive effect on the output voltage value of multifunction calibrator due to many kinds of reasons, and these two variables $\Delta V_{i}$ and $\Delta V_{s}$ generally obey the uniform distribution.

We use the MCM to evaluate the measurement uncertainty of the six-and-a-half digital multimeter, and verify the validity of MCM for evaluating the measurement uncertainty. Fig. 4 shows the experimental environment of DMM measurement uncertainty evaluation. It can be assumed that the reading itself of DMM doesn't introduce errors because the DMM is a digital display, so the standard deviation is 0 . The uncertainty of the multifunction calibrator is given by the instructions or calibration certificate, and when set multifunction calibrator voltage $100 \mathrm{~V}$, the standard deviation of multifunction calibrator is 0.00027 . The PDF of each input and related information are shown in Table 1. In the method of MCM evaluating the DMM uncertainty, the selected sample size is $M=10^{6}$. The measurement result of DMM from 1 to 1000 is shown in 
Fig. 5, and the PDF of the DMM uncertainty is shown in Fig. 6. The mean value of DMM measurement uncertainty is $E_{Y}=5.13 \times 10^{-7}$, and the standard deviation of DMM measurement uncertainty is $S_{Y}=2.72 \times 10^{-4}$. $95 \%$ of the inclusion interval is $\left[-0.53 \times 10^{-3}, 0.53 \times 10^{-3}\right]$. The PDF of the uncertainty of DMM measurement uncertainty is similar to the normal distribution, which can DMM measurement uncertainty $N\left(5.13 \times 10^{-7}, 0.000272^{2}\right)$, so its $95 \%$ of the inclusion interval is symmetric interval. From the evaluation result, the uncertainty of the DMM is small and its measurement result is credible. At the same time, the uncertainty of the DMM measurement result mainly comes from the uncertainty of multifunction calibrator, while the uncertainty of other aspects has little effect on the uncertainty of the measurement result of DMM.

Table 1. PDF of DMM measurement model input and related information

\begin{tabular}{|c|c|c|c|}
\hline Variable & $\begin{array}{l}\text { Distribution } \\
\text { type }\end{array}$ & PDF & $\begin{array}{l}\text { Sensitivity } \\
\text { coefficient }\end{array}$ \\
\hline$V_{i}$ & $\begin{array}{c}\text { Normal } \\
\text { distribution }\end{array}$ & $\mathrm{N}(100,0)$ & 1 \\
\hline$V_{s}$ & $\begin{array}{l}\text { Normal } \\
\text { distribution }\end{array}$ & $\mathrm{N}(100,0.000272)$ & -1 \\
\hline$\Delta V_{i}$ & $\begin{array}{l}\text { Uniform } \\
\text { distribution }\end{array}$ & $\begin{array}{c}\mathrm{R}(- \\
0.00005,0.00005)\end{array}$ & 1 \\
\hline$\Delta V_{s}$ & $\begin{array}{l}\text { Uniform } \\
\text { distribution }\end{array}$ & $\begin{array}{c}\mathrm{R}(- \\
0.000035,0.000035)\end{array}$ & -1 \\
\hline
\end{tabular}

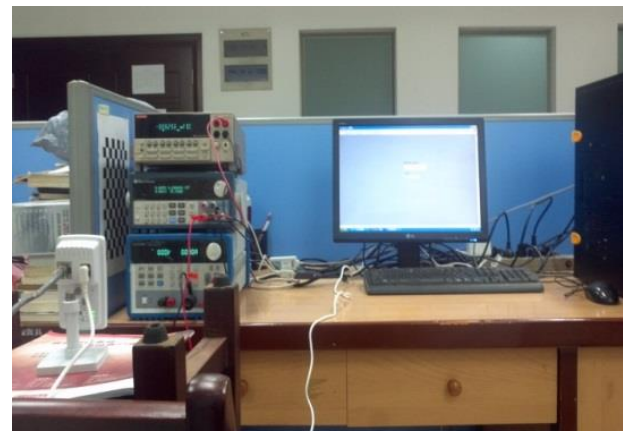

Figure 4. The experimental environment of DMM measurement uncertainty evaluation

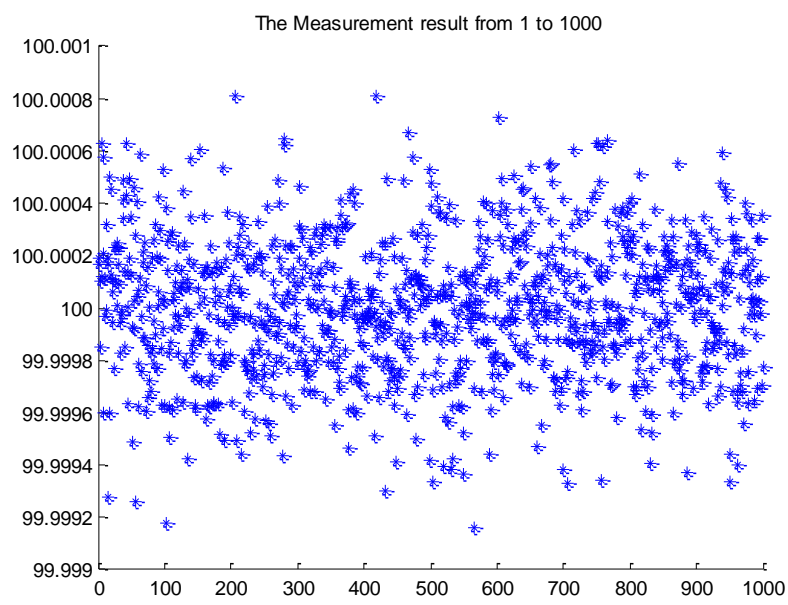

Figure 5. The measurement result of DMM from 1 to 1000

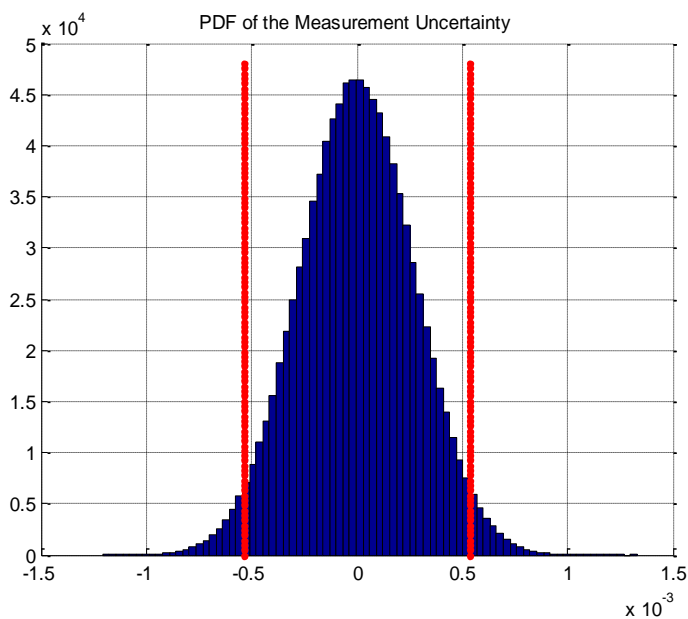

Figure 6. PDF of the DMM uncertainty

\section{Conclusions}

This paper mainly studies the measurement uncertainty evaluation of the digital multimeter by Monte Carlo method. Monte Carlo method has a strong applicability in evaluating measurement uncertainty. The advantages of Monte Carlo method for evaluating measurement uncertainty are analyzed theoretically, and the steps and precautions of Monte Carlo method for evaluating measurement uncertainty are introduced in detail. Finally, the six-and-a-half digital multimeter uncertainty is evaluated by the Monte Carlo method, and the probability density function of digital multimeter uncertainty obeys the normal distribution, which has high precision. The Monte Carlo method used to evaluate the measurement uncertainty can obtain the probability density function of output directly. Therefore, it has a very prominent advantage and operability in the complex nonlinear model.

\section{Acknowledgments}

This work is supported by grants from The Chinese People's Liberation Army General Armament Department. The department of instrument offers the guidance of MCM on DMM.

\section{References}

1. Gen W 2013 Evaluation of measurement error and uncertainty (2th edition) (Beijing: China zhijian publishing house, Standards press of China) pp 77-90

2. BIPM, IEC, IFCC, ISO, IUPAC, IUPAP, OIML 1993 Guide to the expression of uncertainty in measurement (Geneva, Switzerland.

3. General Administration of Quality Supervision, Inspection and Quarantine of the People's Republic of China 2013 Evaluation and expression of uncertainty in measurement (Beijing: China zhijian publishing house)

4. Chen X, Bo X, Wang H 2005 The evaluation of combined uncertainty based on the theory of Monte Carlo Chinese Journal of Scientific Instrument vol 26(z1) pp 759-761 
5. Ge L 2006 Uncertainty evaluation for virtual instrument measurements based on Monte Carlo method (Hefei: HeFei University of Technology) pp 25-37

6. Chen H, Cao Y, Han J 2011 Evaluation of uncertainty in measurement based on a Monte Carlo method Journal of Electronic Measurement and Instrumentation vol 25(4) pp 301-308

7. Wang W, Song M, Chen Y, Gu L, Tao J 2008 Application of Monte-Carlo method in measurement uncertainty evaluation of complicated model Chinese Journal of Scientific Instrument vol 29 pp 1446-1449
8. Wang J, Liu Q, Yang H 2016 Moisture detector standard uncertainty evaluation based on Monte Carlo method World Sci-Tech R \& D vol 2 pp 297-300

9. $\mathrm{Ni}$ Y 2015 Practical evaluation of uncertainty in measurement (5th edition) (Beijing: China zhijian publishing house, Standards press of China) pp 35-54

10. Cao Y, Chen W 2012 Analysis of uncertainty using MATLAB Metrology \& Measurement technique vol39(2) pp 55-56

11. Huang F, Zhou X, Chen G 2016 Uncertainty evaluation of digital multimeter measurement and implementation based on LabVIEW Industrial Metrology vol s2 pp 43-45 